\title{
Efficacy of recombinant human adenovirus-p53 combined with chemotherapy for locally advanced cervical cancer: A clinical trial
}

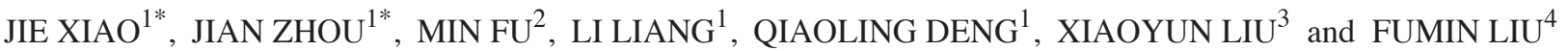 \\ ${ }^{1}$ Department of Gynecology and ${ }^{2}$ Cervical Disease Center, Xuzhou Maternity and Child Health Care Hospital, \\ Xuzhou Medical College; ${ }^{3}$ Center Laboratory and ${ }^{4}$ Department of Obstetrics and Gynecology, \\ Affiliated Hospital of Xuzhou Medical College, Xuzhou, Jiangsu 221002, P.R. China
}

Received July 15, 2016; Accepted February 9, 2017

DOI: $10.3892 / \mathrm{ol} .2017 .5901$

\begin{abstract}
The aim of this study was to evaluate the efficacy and safety of recombinant human adenovirus-p53 (rhAd-p53) combined with neoadjuvant chemotherapy in treatment of locally advanced cervical cancer (LACC). A total of 40 patients with LACC (stage IB2 to IIIA) were randomized into 2 groups $(n=20$ each): PVB group (cisplatin + vincristine + bleomycin, intravenously) and combined group (rhAd-p53 gene therapy + neoadjuvant chemotherapy). Both groups underwent a course of chemotherapy; the only exception was the injection of the rhAd-p53 solution $1 \times 10^{12} \mathrm{VP}$ intratumorally at an interval of three days thrice in the combined group thereafter. The tumor sizes and adverse events in both groups were observed. The expression of vascular endothelial growth factor (VEGF), protein p53 and micro-vessel density (MVD) in tumor tissue was respectively determined by immunohistochemistry. The evaluation was performed three weeks after the completion of chemotherapy. The efficacy was $75 \%$ in the PVB group versus $95 \%$ efficacy in the combined group; the tumor size was reduced by $11.42 \pm 2.78 \mathrm{~cm}^{2}$ in PVB group versus the significant shrinkage of $15.25 \pm 4.00 \mathrm{~cm}^{2}$ in the combined group $(\mathrm{P}<0.05)$. The expression of VEGF, p53 and MVD was downregulated in both the PVB and combined groups, with significantly statistical differences versus the control. No additional adverse events were evidenced in the combined group. Therefore, intratumoral injection of rhAd-p53 combined with neoadjuvant chemotherapy
\end{abstract}

Correspondence to: Dr Fumin Liu, Department of Obstetrics and Gynecology, Affiliated Hospital of Xuzhou Medical College, 99 Huai-hai West Road, Xuzhou, Jiangsu 221002, P.R. China E-mail: liufumin820@163.com

*Contributed equally

Abbreviations: LACC, locally advanced cervical cancer; FIGO, International Federation of Gynecology and Obstetrics; rhAd-p53, recombinant human adenovirus-p53; VEGF, vascular endothelial growth factor; MVD, micro-vessel density; CR, complete response; $\mathrm{PR}$, partial remission; $\mathrm{SD}$, stable disease; $\mathrm{PD}$, progressive disease

Key words: adenoviruses, human, genes, p53, cervical cancer, chemotherapy, combination has advantage over conventional chemotherapy for its high efficacy, safety and synergism in the therapy for LACC.

\section{Introduction}

Locally advanced cervical cancer (LACC), which is classified by International Federation of Gynecology and Obstetrics (FIGO, 2009) as stages IB2-IVA, is a group of high risk cervical cancers with poor prognosis (1). For young patients with LACC, neoadjuvant chemotherapy is now becoming a preferable therapy option prior to radical surgery $(2,3)$. Nevertheless, the systemic adverse reactions due to chemotherapeutics, the low complete remission rate from single chemotherapeutics as well as tumor cell resistance all hamper the efficacy of chemotherapy. Therefore, more effective treatment strategies are still required. Wild-type p53 (wt-p53) gene is an essential cancer suppressor gene. Evidence generated over the past decade has shown that the p53 tumor suppressor is the most frequently altered gene in $>50 \%$ of all types of human cancers. In addition, gene therapy with wt-p53 has emerged as a promising antitumor strategy for some malignant tumors $(4,5)$. Wt-p53 is a 'housekeeping gene' and functions as an inhibitor of carcinogenesis by maintaining genomic integrity; the wt-p53 protein is a primary mediator of cell cycle arrest, DNA repair and apoptosis. The inactivated p53 protein and the aberrant p53 tumor suppressor pathway plays important roles in the pathogenesis of cervical cancer. It would be interesting to reconstruct the p53 pathway by gene therapy. In our previous study, we confirmed that recombinant human adenovirus-p53 (rhAd-p53) could inhibit the proliferation of HeLa cells in vitro and the p53 agent combined with chemotherapeutics significantly inhibited cancer cell proliferation and effectively induced apoptosis (6). The p53 agent potentiated the sensitivity of cancer cells to chemotherapy. However, there has been a paucity of reports on the clinical applications of p53 agent to cervical cancer. In this study, rhAd-p53 was intratumorally injected into LACC in patients of FIGO stages IB2-IIIA, coupled with PVB chemotherapy, which may be able to provide a novel clinical regimen for LACC.

\section{Materials and methods}

Patients and tissues. A total of 40 patients with cervical cancer of FIGO stages IB2-IIIA hospitalized in the Maternity 
and Child Health Care Hospital Affiliated to Xuzhou Medical College (Xuzhou, China) were recruited. Experimental protocol was approved by the hospital Ethics Committee, and written informed consent was obtained from each patient. Inclusion criteria: Premenopausal patients aged $\leq 45$ years, cervical squamous cell carcinoma diagnosed by cervix biopsy, expected survival of at least six months, lesions clinically measurable and feasible for drug injection, no prior chemotherapy and/or radiotherapy, normal functions of heart, lungs, liver and kidneys, void of contraindications to chemotherapy.

A total of 40 patients were randomized into 2 groups $(n=20$ each): PVB group (cisplatin + vincristine + bleomycin, intravenously) and combined group (rhAd-p53 gene therapy + PVB chemotherapy). Both groups underwent chemotherapy, the only exception was the administration of the rhAd-p53 solution $1 \times 10^{12}$ VP intratumorally at an interval of 3 days thrice in the combined group.

According to the 2009 amendments to the clinical staging criteria by FIGO, the 40 patients were classified IB2 (6 cases), stage IIA (13 cases), stage IIB (18 cases) and stage IIIA ( 3 cases). The PVB group was aged from 28 to 45 years (mean age, 40 years); their FIGO clinical staging was IB2 (4 cases), IIA (10 cases), IIB (6 cases) and IIIA (0 case); their pre-treatment tumor size was averaged at $19.91 \pm 5.40 \mathrm{~cm}^{2}$. The combined group was aged from 32 to 45 years (mean age, 42 years); their FIGO clinical staging was IB2 (2 cases), IIA 3 cases, IIB (12 cases), IIIA (3 cases); their pre-treatment tumor size was averaged at $22.76 \pm 6.02 \mathrm{~cm}^{2}$. There were no significant differences in age and FIGO clinical staging $(\mathrm{P}>0.05)$ or in pre-treatment tumor size $(\mathrm{t}=1.573, \mathrm{P}=0.124)$ between the two groups.

Tissue samples were obtained from patients in both the PVB and combined group ( $\mathrm{n}=20$ each) that were undergoing tumor resection. A total of 18 samples were obtained from control patients with cervical cancer who did not undergo chemotherapy, radiotherapy or gene therapy.

Regimens and efficacy assessments. rhAd-p53 injections ( $1 \times 10^{12}$ VP/ampule) (Shenzhen SiBiono GeneTech Co., Ltd., Shenzhen, China), approved by the China Food and Drug Administration were stored at $-20^{\circ} \mathrm{C}$ prior to the experiment. Both groups were administered a chemotherapy regimen that comprised of cisplatin, vincristine and bleomycin for 3 consecutive days, whereas the combined group proceeded to receive multiple intratumoral injection of rhAd-p53 solution $1 \times 10^{12} \mathrm{VP}$ at an interval of three days thrice after end of chemotherapy. For tumors $<4 \mathrm{~cm}$ in diameter, rhAd-p53 solution was diluted to $2 \mathrm{ml}$ by addition of normal saline, and for tumors $>4 \mathrm{~cm}$ in diameter, rhAd-p53 was diluted to $4 \mathrm{ml}$. Efficacy was assessed 3 weeks thereafter. Tumor sizes were compared prior and subsequent treatment consistently by ultrasonography. Tumor size is defined as mass product of the two greatest perpendicular diameters. In conformity with the WHO evaluation of efficacy of solid tumors of complete response (CR), partial remission $(\mathrm{PR})$, stable disease (SD), progressive disease (PD), our curative efficiencies $\mathrm{CR}$ and PR were considered effective, whereas SD and PD were null. Moreover, adverse events were observed in all patients throughout the treatment.

Immunohistochemistry. The expression of vascular endothelial growth factor (VEGF), mutant p53 protein and micro-vessel density (MVD) in tumors was determined before and after treatment by immunohistochemistry using VEGF and CD34 reagents (Boster Biological Technology Co. Ltd., Wuhan, China) and the p53 protein detection reagent (Beijing Zhongshan Golden Bridge Biotechnology Co., Ltd., Beijing, China). Positive staining of VEGF was demonstrated by the presence of brown-yellow granules in the cytoplasm, and positive staining of p53 denoted tan-colored nuclei.

Semi-quantitative immunohistochemical scoring: i) Positive cell scoring: 0 , for proportion of positive cells below $1 \%$; 1 , for $1-10 \%$; 2 , for $11-50 \%$; 3 , for $51-80 \%$; and 4 , for $>80 \%$; ii) staining intensity scoring: 1 , for weak staining; 2 , for moderate staining; and 3 , for strong staining. The sum of two items represented the eventual result: $1,(-) ; 2-3,(+)$; 4-5, (++); and 6-7, (+++). CD34 stain is claybank located on vascular endothelial cell membrane of the tumor and stroma. Microvessel count criteria: any single endothelial cell or endothelial cell clusters that are CD34 dyed brown are regarded as a microvessel as long as they are separated from the adjacent microvessels, tumor cells or other connective tissue. Similarly, it is a vessel if the structure is not connected to its branching structure.

Routine clinical examination and management of adverse events. Routine tests of blood, urine and stools, as well as blood biochemistry, ECG and chest X-ray were conducted at intervals and coupled with surveillance of gastrointestinal tract, hepatic functionalities, temperature variations and signs of bone marrow suppression. A few hours after the rhAd-p53 injection, patients may experience a self-limited temporary fever, for which antipyretic (indomethacin suppository) could be administered if needed.

Statistical analysis. Statistical analysis was performed using the statistical software SPSS 12.0 (SPSS, Inc., Chicago, IL, USA). MVD counts and tumor size before and after treatment are shown as mean \pm standard deviation. Post-therapeutic focal shrinkage was compared using t-test and comparison of the efficacies between the groups was performed with the rank sum test and Spearman correlation test. Adverse events and expression of VEGF and p53 between groups was compared and analyzed by $\chi^{2}$ test. The MVD counts between groups were analyzed with one-way analysis of variance. $\mathrm{P}<0.05$ was considered to indicate a statistically significant difference.

\section{Results}

Therapeutic efficacy. Post-treatment cancer foci were reduced to $11.42 \pm 2.78$ and $15.25 \pm 4.00 \mathrm{~cm}^{2}$ in the PVB group and the combined group, respectively $(\mathrm{t}=3.504, \mathrm{P}=0.001)$. The total efficacy rate in the PVB group which consisted of $0 \mathrm{CR}$, $15 \mathrm{PR}, 5 \mathrm{SD}$ and $0 \mathrm{PD}$ was $75 \%$, whereas that in combined group comprised of $2 \mathrm{CR}, 17 \mathrm{PR}, 1 \mathrm{SD}$ and $0 \mathrm{PD}$ was $95 \%$; the results were statistically significant $(\mathrm{Z}=2.137, \mathrm{P}=0.033)$.

Detection of VEGF, mutant 553 protein and MVD in tumor tissues. The expression of VEGF in control, PVB group and rhAd-p53 + PVB group was 88.9 (16/18), 80.0 (16/20) and $50.0 \%(10 / 20)$, respectively; results were statistically significant $(\mathrm{P}<0.05)$ (Fig. 1A-C). The expression of the mutant $\mathrm{p} 53$ protein in each of the three groups was $83(15 / 18), 60(12 / 20)$ 

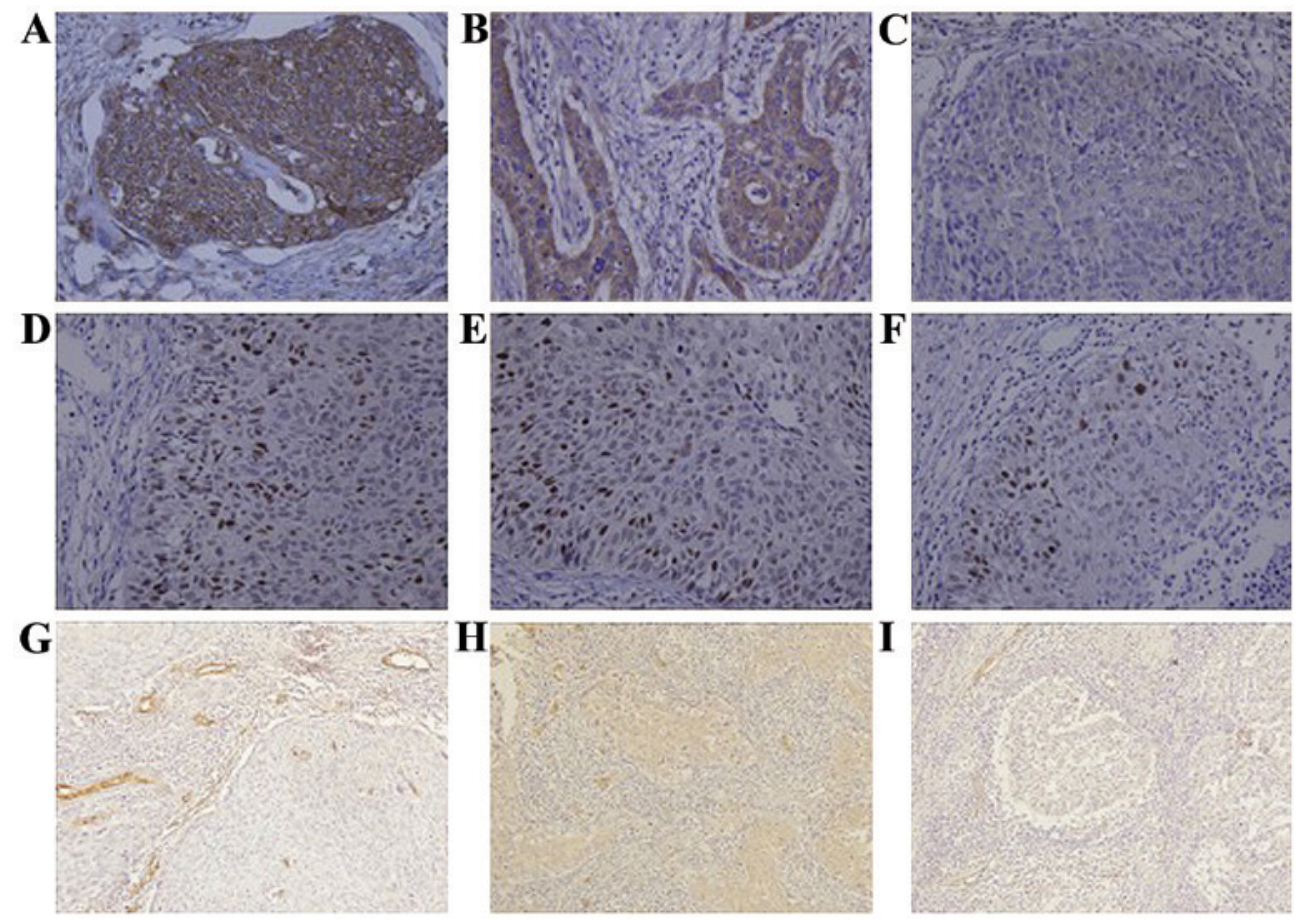

Figure 1. The expression of VEGF, p53 protein and MVD in cervical cancer tissue (SP). (A-C) The expression of VEGF in control, PVB group and rhAd-p53 + PVB group (magnification, x400); (D-F) The expression of mutant p53 protein in control, PVB group and rhAd-p53 + PVB group (magnification, x400); (G-I) MVD in control, PVB group and rhAd-p53 + PVB group (magnification, x200). VEGF, vascular endothelial growth factor; MVD, micro-vessel density.

and 25\% (5/20), respectively; results were statistically significant $(\mathrm{P}<0.05)$ (Fig. 1D-F). MVD in the three groups counted for $64.7 \pm 2.3,42.5 \pm 2.2$ and $32.5 \pm 1.3$, respectively; results were statistically significant $(\mathrm{P}<0.05$; Fig. 1$)$.

Adverse reactions. Both groups suffered from adverse reactions, mainly gastrointestinal reactions, bone marrow suppression, liver damage and fever. In the PVB group, 15 cases of gastrointestinal discomfort such as nausea and vomiting were observed, 3 patients had second-degree bone marrow suppression and 1 patient had third-degree bone marrow suppression. All were given recombinant human granulocyte colony-stimulating factors to support symptoms improvement after treatment. No patient showed signs of infection. Three patients had elevated aminotransferase levels, which improved after being treated for liver disease. Ten patients presented with fever, which improved after symptomatic treatment. In rhAd-p53 + PVB group, 13 cases of patients had gastrointestinal discomfort, which was tolerated. One patient had bone marrow suppression and was given recombinant human granulocyte colony-stimulating factor to support symptoms improved after treatment. Two patients who had elevated aminotransferase levels improved after being given liver treatment. Fifteen patients that were given the rhAd-p53 injection had fever, among which 10 had a temperature of $38^{\circ} \mathrm{C}$, which returned to normal 12-24 $\mathrm{h}$ after drinking a lot of water; the other 5 had a temperature of $39^{\circ} \mathrm{C}$, which returned to normal 12-24 h after indomethacin anal plug symptomatic treatment. None of the patients showed signs of upper respiratory tract infection. There was no statistically significant difference in fever, gastrointestinal reactions, bone marrow suppression and liver damage rate $(\mathrm{P}>0.05)$.

\section{Discussion}

rhAd-p53 is a replication-defective living adenovirus carrying the p53 gene. The advantage of the adenovirus delivery system is that it does not result in integration of the vector DNA into the host cells (7). Once cells have been infected, virus particles cannot breed, while it can import the p53 gene, which exerted biological functions. DNA damage within cancer cells is the important internal factor which triggers the p53 gene and (or) p53 protein responses. Basic mechanism of rhAd-p53 reagents are: i) Inhibit tumor growth by cell cycle arrest and induce 'programmed cell death'; ii) enhance chemotherapy-induced cell cycle arrest and apoptosis; iii) stimulate the body to produce anti-tumor immune response in a way that the local injection site of tumors gather a large number of immune cells; iv) inhibit tumor VEGF to suppress angiogenesis and tumor growth through the 'bystander effect' (8), so the injection site of the local tumor tissue will block blood supply and induce tumor necrosis.

The basic theory for gene therapy is completely different from that of the traditional treatment such as chemotherapy and radiotherapy; in fact, gene therapy and chemotherapy could complement each other (9-11). Our previous study found that rhAd-p53 can inhibit the proliferation of cervical cancer (HeLa) cells in vitro; furthermore, a p53 agent combined with chemotherapeutics leads to a greater inhibition in cell proliferation and a higher apoptosis rate (8). Some studies have also shown that gene therapy in combination with chemotherapy leads to a beneficial effect for head and neck squamous cell carcinoma, lung cancer and gastric cancer (12-14).

In this study, we combine gene therapy and chemotherapy for the treatment of LACC and found that the PVB group 
and rhAd-p53 + PVB group can shrink the tumor volume. Moreover, the shrunken volume is 1.34 times higher in rhAd-p53 + PVB group than the PVB group alone, and the efficiency of the rhAd-p53 + PVB group is 1.27 times that of PVB group. Therefore, rhAd-p53 has a significant synergistic effect with cervical cancer chemotherapy.

VEGF is the most critical growth factor to stimulate tumor angiogenesis (15), which specifically targets vascular endothelium to promote endothelial cell proliferation and migration. It favors the formation of new blood vessels and increases the permeability of existing blood vessels (16). Consequently, it contributes to the growth, invasion and metastasis of tumor cells (17). The wt-p53 expression was inversely associated with VEGF expression, suggesting that wt-p53 is involved in the suppression of the VEGF gene (18). Some researchers (19) reported that a downregulation of VEGF was observed in tumor biopsy tissue treated with an intratumoral injection of rhAd-p53. Significant necrosis was found in tumor biopsy tissues of patients who received intratumoral injections of rhAd-p53, which may be correlated with rhAd-p53-induced downregulation of VEGF. In our study, the expression of VEGF in the PVB group and the rhAd-p53 + PVB group was significantly decreased compared to the control. In addition, the rhAd-p53 + PVB group had a more significant decrease in VEGF than the PVB group. Hence, the expression of VEGF is effectively decreased when gene therapy is combined with chemotherapy, which inhibits the formation of angiogenesis in cervical cancer.

The expression of the p53 gene in cervical cancer was significantly decreased (20), and the inactivation of p53 gene led to an increase in the invasion of cervical cancer (21). The mutant p53 protein gathers in the cell nucleus and the half-life period is significantly extended, which helps us detect abnormal expression of the p53 protein. In our study, the expression of the mutant p53 protein in PVB and rhAd-p53 + PVB group was significantly decreased when compared to the control, and rhAd-p53 + PVB was decreased much more than the PVB group. The above results suggest that the mutant $\mathrm{p} 53$ protein enhances the transcription and expression of VEGF (22). rhAd-p53 reagents can effectively inhibit the expression of mutant p53 protein. Also, they inhibit tumor angiogenesis by suppressing the transcription and expression of VEGF.

The formation of blood vessels accelerated the growth and metastasis of tumor cells, and MVD in the tumor tissue reflected the malignancy and invasiveness of the tumors. The CD34 antigen, a highly glycosylated type I transmembrane protein with a relative molecular mass of 90x103-120x103, is selectively expressed on the surface of human vascular endothelial cells. CD34 is an antigen that is related to new small blood vessels, which is a more specific marker of mature vascular endothelial cells. We used CD34 antigens as markers to count microvessels, which can reflect tumor microvessel quantity accurately and objectively (23). In our study, the MVD count in PVB and rhAd-p53 + PVB group is significantly decreased, with the latter demonstrating a more considerable decrease. This result is consistent with a decrease in mutated p53 protein and VEGF, which suggests that gene therapy, in combination with chemotherapy, can be more effective in inhibiting tumor angiogenesis and limiting the growth of tumors.
In this study, the rhAd-p53 reagent caused a self-limiting fever in patients. Febrile reactions mainly occured several hours after the injection and few on the following day. Symptoms eased without obvious discomfort after drinking more water or the use of antipyretic drugs, which does not affect subsequent treatment. There was no statistical significance in fever, gastrointestinal reactions, bone marrow suppression and liver damage between the PVB group and rhAd-p53 + PVB group, which tells us that gene dosage does not have serious side effects.

Through this study, we realized that rhAd-p53 can effectively inhibit the expression of the mutant p53 protein, and inhibit the transcription and expression of VEGF once it gets into tumor cells. This reduces tumor angiogenesis and is more effective in shrinking tumor volume after interaction with chemotherapeutic drugs. Furthermore, it provides conditions for surgical treatment by reducing tumor staging for patients with advanced cervical cancer. There were no other adverse reactions except, for a brief period of time, self-limiting heating during the administration of rhAd-p53 reagent, which suggests that, in addition to its anti-cancer effect, rhAd-p53 reagent can increase sensitivity to chemotherapy for cervical cancer. Therefore, the rhAd-p53 reagent, when combined with chemotherapy drugs, is effective for cervical cancer patients.

\section{Acknowledgements}

This study was supported by Clinical Cancer Research Fund of Health Bureau of Jiangsu Province (no. P200943). We are grateful to Ms. Yao Qier, our professional nurse; her dedication is outstanding and invaluable.

\section{References}

1. Pecorelli S: Revised FIGO staging for carcinoma of the vulva, cervix, and endometrium. Int J Gynaecol Obstet 105: 103-104, 2009.

2. Rydzewska L, Tierney J, Vale CL and Symonds PR: Neoadjuvant chemotherapy plus surgery versus surgery for cervical cancer. Cochrane Database Syst Rev 12: CD007406, 2012.

3. Gadducci A, Sartori E, Maggino T, Zola P, Cosio S, Zizioli V, Lapresa M, Piovano E and Landoni F: Pathological response on surgical samples is an independent prognostic variable for patients with Stage Ib2-IIb cervical cancer treated with neoadjuvant chemotherapy and radical hysterectomy: An Italian multicenter retrospective study (CTF Study). Gynecol Oncol 131: 640-644, 2013.

4. Nemunaitis J, Clayman G, Agarwala SS, Hrushesky W, Wells JR, Moore C, Hamm J, Yoo G, Baselga J, Murphy BA, et al: Biomarkers predict p53 gene therapy efficacy in recurrent squamous cell carcinoma of the head and neck. Clin Cancer Res 15: 7719-7725, 2009.

5. Lehmann BD and Pietenpol JA: Targeting mutant p53 in human tumors. J Clin Oncol 30: 3648-3650, 2012.

6. Zheng XL, Wang TT, Fu M, Zhou J and Liu FM: Inhibitory effect of recombinant human adenovirus-P53 combined with paclitaxel on human cervical cancer HeLa cells and its mechanism. Chin J Cancer Biother. 20: 192-196, 2013 (In Chinese).

7. Lane DP, Cheok CF and Lain S: p53-based cancer therapy. Cold Spring Harb Perspect Biol 2: a001222, 2010.

8. Burdak-Rothkamm S, Rothkamm K, McClelland K, Al Rashid ST and Prise KM: BRCA1, FANCD2 and Chk1 are potential molecular targets for the modulation of a radiation-induced DNA damage response in bystander cells. Cancer Lett 356 (Pt B 2): 454-461, 2015

9. Jiang G, Xin Y, Zheng JN and Liu YQ: Combining conditionally replicating adenovirus-mediated gene therapy with chemotherapy: A novel antitumor approach. Int J Cancer 129: 263-274, 2011. 
10. Prados J, Alvarez PJ, Melguizo C, Rodriguez-Serrano F Carrillo E, Boulaiz H, Vélez C, Marchal JA, Caba O, Ortiz R, et al: How is gene transfection able to improve current chemotherapy? The role of combined therapy in cancer treatment. Curr Med Chem 19: 1870-1888, 2012.

11. Wirth T, Parker N and Ylä-Herttuala S: History of gene therapy. Gene 525: 162-169, 2013.

12. Tassone P, Old M, Teknos TN and Pan Q: p53-based therapeutics for head and neck squamous cell carcinoma. Oral Oncol 49: 733-737, 2013

13. Li D, Zhang Y, Xie Y, Xiang J, Zhu Y and Yang J: Enhanced tumor suppression by adenoviral PTEN gene therapy combined with cisplatin chemotherapy in small-cell lung cancer. Cancer Gene Ther 20: 251-259, 2013.

14. Chen GX, Zheng LH, Liu SY and He XH: rAd-p53 enhances the sensitivity of human gastric cancer cells to chemotherapy. World J Gastroenterol 17: 4289-4297, 2011.

15. Cao Y, Guangqi E, Wang E, Pal K, Dutta SK, Bar-Sagi D and Mukhopadhyay D: VEGF exerts an angiogenesis-independent function in cancer cells to promote their malignant progression. Cancer Res 72: 3912-3918, 2012.

16. Tomao F, Papa A, Rossi L, Zaccarelli E, Caruso D, Zoratto F, Benedetti Panici P and Tomao S: Angiogenesis and antiangiogenic agents in cervical cancer. Onco Targets Ther 7: 2237-2248, 2014.
17. Perrot-Applanat $\mathrm{M}$ and Di Benedetto $\mathrm{M}$ : Autocrine functions of VEGF in breast tumor cells: Adhesion, survival, migration and invasion. Cell Adhes Migr 6: 547-553, 2012.

18. Holmgren L, Jackson $G$ and Arbiser J: p53 induces angiogenesis-restricted dormancy in a mouse fibrosarcoma. Oncogene 17: 819-824, 1998.

19. Li X, Xiao S, Li Y and Zhang S: Clinical antiangiogenic effect of recombinant adenovirus-p53 combined with hyperthermia for advanced cancer. Chin J Cancer Res 25: 749-755, 2013.

20. Crinelli R, Bianchi M, Menotta M, Carloni E, Giacomini E, Pennati $\mathrm{M}$ and Magnani M: Ubiquitin over-expression promotes E6AP autodegradation and reactivation of the p53/MDM2 pathway in HeLa cells. Mol Cell Biochem 318: 129-145, 2008.

21. Yang $X$ and Lu L: Expression of HPV-16 E6 protein and p53 inactivation increases the uterine cervical cancer invasion. Drug Res (Stuttg) 65: 70-73, 2015.

22. Yu YF, Zhang Y, Shen N, Zhang RY and Lu XQ: Effect of VEGF, P53 and telomerase on angiogenesis of gastric carcinoma tissue. Asian Pac J Trop Med 7: 293-296, 2014.

23. Ding S, Li C, Lin S, Yang Y, Liu D, Han Y, Zhang Y, Li L, Zhou L and Kumar S: Comparative evaluation of microvessel density determined by CD34 or CD105 in benign and malignant gastric lesions. Hum Pathol 37: 861-866, 2006. 